\title{
Artificial Neural Network Model in Spatial Analysis of Geographic Information System
}

\author{
Shaofu $\mathrm{He}^{1}$ and Fei Li iD $^{2,3}$ \\ ${ }^{1}$ Minnan Normal University, Zhangzhou 363000, Fujian, China \\ ${ }^{2}$ Institute of Geographic Sciences and Natural Resources Research, Chinese Academy of Sciences, Beijing 100101, China \\ ${ }^{3}$ University of Chinese Academy of Sciences, Beijing 100049, China \\ Correspondence should be addressed to Fei Li; lifeicas@126.com
}

Received 27 August 2021; Revised 15 September 2021; Accepted 29 September 2021; Published 15 October 2021

Academic Editor: Sang-Bing Tsai

Copyright (c) 2021 Shaofu He and Fei Li. This is an open access article distributed under the Creative Commons Attribution License, which permits unrestricted use, distribution, and reproduction in any medium, provided the original work is properly cited.

\begin{abstract}
In the past two decades, the computer technology industry has developed rapidly, and the geological prospecting industry is also undergoing a computerized and electronic revolution. The application technology of new geological information systems is gradually adding us to the spatial information system of geological prospecting projects. In order to deeply study the current situation of the artificial neural network model in the spatial analysis of our country's geographic information system, this paper uses the traditional classification analysis method; database analysis and neural network analysis method of compensating samples were collected, an artificial model of the network is established, and the algorithm is simplified. And a neural network model is created. In the research of A and B counties' geographic information system, using a new network model, 61 geological disasters were found in County A, of which 47 were landslides, 4 collapses, and 10 unstable slopes. There were 19 geographical disasters in County B, including 9 unstable slopes, 6 landslides and 4 collapses. In terms of geographic prediction combined with the network model, the comparison with the actual situation shows that the geographical distribution is $99.7 \%$ in the geographical and geological disaster-prone areas, and the geographical distribution is less in the nonprone areas, with a proportion of $0.3 \%$. Geological disaster-prone areas of low points accounted for $76.9 \%$, and the number of disaster-affected points in the low-prone areas accounted for $22.8 \%$. The geographical and geological grades divided by the evaluation model are basically consistent with the actual grades, which can meet the needs of geographic evaluation. It is basically realized that starting from the model's geographic information system, a more comprehensive and practical artificial neural network model is designed.
\end{abstract}

\section{Introduction}

Prior to the rapid development of computer science and technology, geographic information has very limited application, and it was generally regarded as the scientific application of natural environment, surveying, drawing, water conservancy, and other disciplines. Now we all know that geographic information system has been applied to all aspects of society, so what exactly is the geography information system? The geography information system is a computer-based tool to solve various geological problems. Geographic Information System helps us understand and find ways to solve difficult problems by collecting information related to geography. For various objects around, such as rivers, streets, residential areas, etc., the geographic information system stores them in the database in the form of data and dynamically links them to the map. When the actual object changes, the data associated with it in the database should also be sent and changed, and the output mapping will be updated accordingly with the database.

As an important component of various geological exploration databases, the current application is mainly for the large number of initial flows of geological exploration-related work to drain into the database and perform corresponding work in the database, while ensuring the absolute safety of the data in the database. In this way, the storage rate 
of the project can be improved, the progress of the project can be grasped in time, the quality is guaranteed, the inspection and clearance during the project completion can be further strengthened, and the problems existing in the project development process can be corrected. With the continuous improvement and maturity of the system functions of the geological prospecting supervision platform, the data storage module will further realize and improve the data entry and management functions for the two types of data existing in the process of geological prospecting projects: spatial data sets and nonspatial data sets. The function of the data storage module is applied to handheld devices, making it more convenient for geologists to use it in the field and providing better services for geological prospecting work.

In 2018, based on the characteristics of groundwater pollution in typical research areas, $\mathrm{Li}$ first selected the main control factors for risk assessment of karst groundwater pollution in mountainous areas. On this basis, the method of quantitative factors was determined, and the risk evaluation index system of karst groundwater pollution was established. In order to overcome the shortcomings of the method of determining factor weights in traditional evaluation methods, Li determines the structure of the artificial neural network model by combining selected evaluation factors. And the weight coefficient of each layer of evaluation factors is calculated. On this basis, a risk assessment model for karst groundwater pollution was established. And the geographic information system to superimpose the weight of each sublayer was used, and the groundwater pollution risk zoning evaluation map of the typical research area was made. The method he adopted can comprehensively and objectively reflect the fact that groundwater pollution is controlled by multiple factors and reveal the nonlinear characteristics of the pollution process. However, his research focuses on building models rather than optimizing algorithms. The proposed algorithm is still not easy to understand and operate [1]. In 2016, Arabgol provided a set of easily measurable groundwater quality variables (including water temperature, conductivity, groundwater depth, total dissolved solids, dissolved oxygen, $\mathrm{pH}$, land use, and seasons of the year) as input variables to predict nitric acid salt concentration tool. The data set includes 160 water samples, representing 40 different wells, and monitored for 1 year. The relevant parameters of the best SVM model are obtained using a combination of 4 -fold cross-validation and grid search technology. The optimal model is used to predict the concentration of nitrate in the aquifer of Arak Plain. The SVM model predicts that the nitrate concentration in the data set during the training and testing phases has a fairly high correlation with the measured value (0.92 and 0.87 , resp.), and the root mean square error is 0.086 and 0.111 , respectively. Finally, a trained SVM model and a geographic information system (GIS) interpolation scheme were used to map the nitrate concentration in groundwater for all four seasons, and the results were compared with a physical-based (flow and pollutant) model. Overall, the results show that the SVM model can be used as a fast, reliable, and cost-effective method for evaluating and predicting groundwater quality. However, there are errors in his research process, leading to deviations in the results [2]. In 2016, Ilia determined the location of the landslide through field investigations and interpretation of aerial photos, thereby constructing a list map containing 132 landslide events, and at the same time determined and used 8 influencing variables. All variables related to the landslide are converted into a $5 \times 5$-meter floating-point raster file. These input raster layers include lithology unit layer, elevation layer, slope angle layer, aspect layer, distance from structural feature layer, distance from hydrological network layer, topographic moisture index layer, and curvature layer. Validation of the developed model was achieved by using a subset of unprocessed landslide data, showing a satisfactory agreement between the expected and existing landslide sensitivity levels, and the area under the predicted rate curve is estimated to be 0.808 . The area under the success rate curve is estimated to be 0.828 , indicating that the classification rate of the existing landslide area is very high. According to the results of sensitivity analysis, the "yellowgray-to-white marl" lithology unit is the most sensitive, and the relative frequency of its landslide changes the most. The overall results of the analysis performed provide key knowledge for successful land use planning and management practices and risk reduction projects. However, he only sampled and collected data on the landslide, and the result was too small and unrepresentative, lacking comprehensiveness [3].

The innovations of this article are as follows: (1) The development history and status quo of artificial neural networks are analyzed [4]. The two unsupervised learning network algorithms are analyzed and researched, and the two calculation processes are tested. (2) The accuracy of the model is improved by using geological information to calculate the marriage, and the improved algorithm reduces The predictability of the data sequence improves the classification effect of the network. (3) Finally, through experiments and mutual comparisons, efficient classification can be achieved, reducing the number of interactions, simplifying adaptive calculations, simplifying algorithms, and improving classification accuracy and output data efficiency [5]. Through the above work, the safety and reliability of the artificial neural network model in the spatial analysis of geographic information systems have been improved [6].

\section{Design and Realization Method Based on the Research of Artificial Neural Network Model in Geospatial Analysis}

2.1. Artificial Neural Network. Artificial neural network (ANN) is based on the cognitive foundation of medical neurosurgery of the human nervous system, exploring the use of computer systems to simulate the human nervous system so that the computer system can be constructed like a nervous system [7]. The human brain can perform working 
memory and processing. If a network system can process things like the human brain, then it becomes a processing process similar to the human brain. The artificial intelligence consists of a large number of simple small cells [8]. It is the reproduction and purpose of the system. Among a large number of factors in various parts, a large number of units are widely produced. It is a general network of the human brain, formed by experiments and simulations, and it represents the characteristics of the human brain. It is similar to the human brain in two ways: strong self-regulation ability, able to learn and train, and gain knowledge through training; information can be learned knowledge (synaptic weights) stored in internal neurons.

As early as the early twentieth century, scientists had to imitate the brain tissue structure model and design a new structure model processed by computer, and storage and processing methods and adopt storage and processing methods that are closer to artificial nerves, solving experimental methods and actual status. It can greatly improve the operating efficiency of the computer, so it is proposed in [9]. In the 1980s and 1990s, the network once again achieved considerable development, with powerful efficiency, and simplification of any algorithm, and the efficiency of related algorithm processes was greatly improved [10].

\subsection{Geographic Information System. Geographical infor-} mation system is a flowchart for collecting, managing, analyzing, and displaying related geographic paths in the whole or part of the land space under the support of contemporary science and technology [11]. Around the 1960s, a country developed the world's first geographic information system in order to facilitate the collection of site geological composition [12]. In the late 1960s, the United States launched a census plan, which realized the sampling and analysis of data from various parts of the country, and it was also used for road and communication management. It has taken the initial form of a contemporary information system. In the 1980s and 1990s, various types of geological systems began to appear. With the maturity of science and technology, the rapid progress of collection, and the gradual improvement of scientific and technological theories, the system technology has gradually matured and is gradually being recognized and accepted by people. Geographical information system has a large number of professional research methods, which are related links for geological analysis. It can combine the spatial geographic location of various objects with corresponding views, reorganize the information according to the needs of users, and obtain satisfactory results for users through spatial analysis tools such as data mining, network analysis, and buffer analysis. It is scientific, intuitive, realtime, and diverse in functions. It is an efficient decisionmaking method [13].

2.3. Network Model. The network model is formed by a large number of neurons connected to each other. Although the structure of the neuron is more complicated, the function it realizes is very simple [14]. The reason why artificial neural networks have strong interaction capabilities is that they are composed of many structural units according to certain interactive connections [15]. According to the past categories of artificial neural networks, artificial neural networks are divided into two types: neural networks that interact with themselves and neural networks that have no interaction with themselves. The two types of artificial neural networks mentioned above will be introduced below.

\subsubsection{Noninteractive Neural Network. Noninteractive neu-} ral network is a kind of neural network without feedback. It has a forward structure and a mutual combination structure, and has an obvious layered structure. Therefore, from the appearance, it is composed of several identical layers and there is no interconnection between them. Network composition: the signal from entering the interaction layer to the end layer is connected in a single direction. Neurons are connected from the starting layer to the next layer [16]. Each neuron only accepts the output of the neuron in the previous layer as input and outputs it to the unit below. There is no connection between the same neurons. An example of a noninteractive neural network is shown in Figure 1 [17]. This article is about the most widely used noninteractive neural network.

As can be seen from Figure 1, A is the noninteractive starting value, $\mathrm{B}$ is the average between the starting layer node and the intermediate value, $C$ is the weight between the intermediate layer node and the final layer node, and D is the final layer node, the last value.

2.3.2. Interactive Neural Network. Interactive neural network is an interactive model, as shown in Figure 2 [18]. The interactive neural network also includes the initial value, the intermediate value, and the result value. Each unit simultaneously uses its own result value as an input to interact with other units, and it takes a period of work to reach stability. If the total number of neurons is $M$, then each neuron has $M$ inputs and one output; that is, each neuron must be connected with other neurons [19]. Its starting value is $\mathrm{A}$, the average number between the starting layer node and the middle layer is $\mathrm{B}$, and the subsequent result value is D. The most widely used interactive neural network is a neural network site [20].

According to Figure 2 we can derive the algorithm steps and mathematical derivation of the BP neural network. The BP neural network algorithm is divided into nine steps:

First, make the network general: take $m$ arbitrary values in $(-1,10)$ and copy these arbitrary values to the interaction value between each layer, set the error function $d$, and determine the exact value $\lambda$ and the maximum number of learning $W[21]$.

Secondly, choose a starting value and its corresponding predicted value in the collected data arbitrarily:

$$
\begin{gathered}
a(n)=\left(a_{1}(n), a_{2}(n), \ldots, a_{m}(n)\right) \\
p_{i}(n)=\left(p_{1}(n), p_{2}(n), \ldots, p_{o}(n)\right) .
\end{gathered}
$$




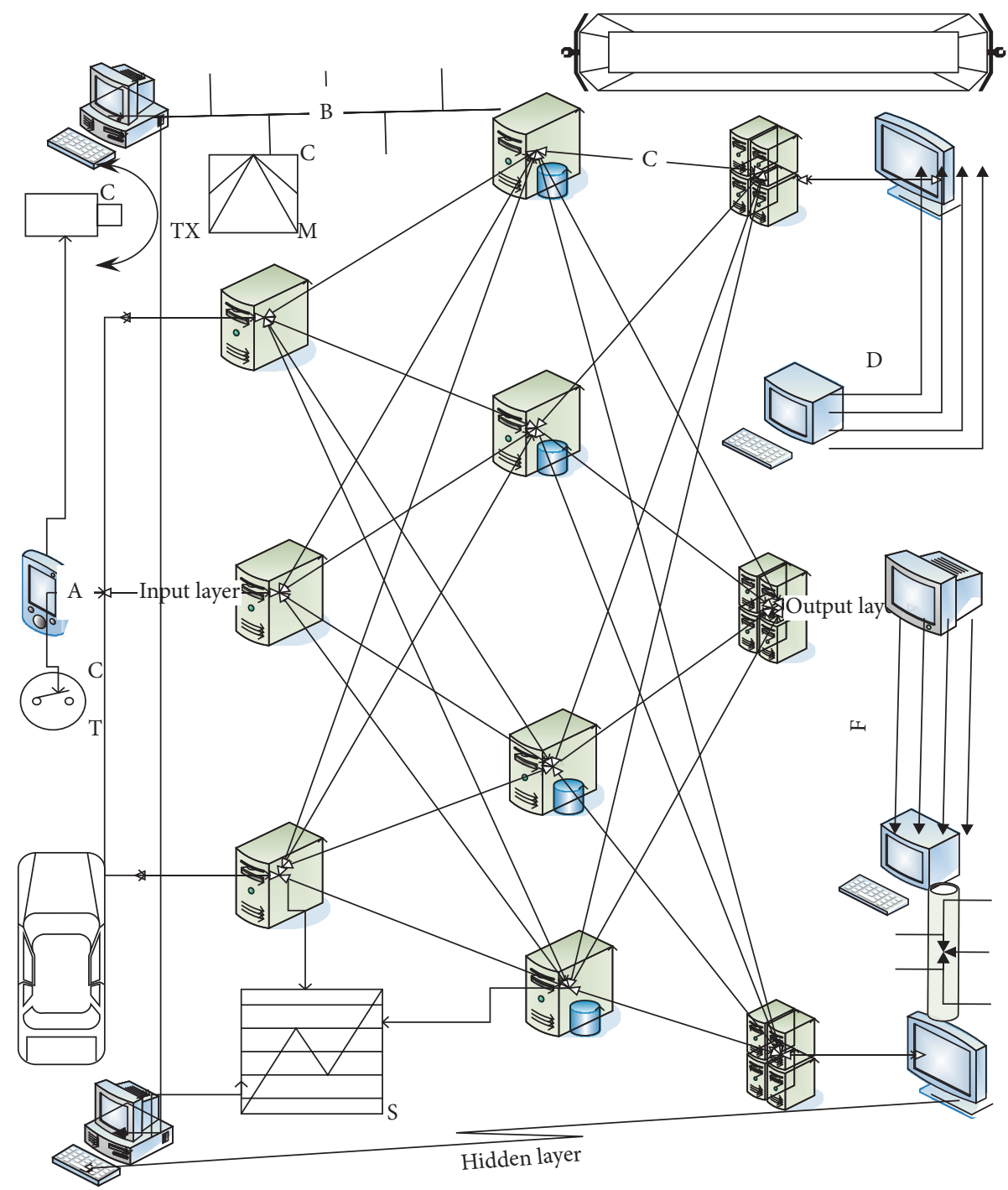

FIGURE 1: Forward neural network.

According to the mathematical formula, calculate the input and output of the hidden layer:

$$
\begin{aligned}
& k c_{k}(n)=\sum_{c=1}^{m} v_{c k} a_{c}(n)-e_{k} \quad k=1,2, \ldots, b, \\
& k u_{k}(n)=s\left(k c_{k}(n)\right) \quad k=1,2, \ldots, b, \\
& x c_{u}(n)=\sum_{k=1}^{b} v_{k u} k u_{k}(n)-e_{u} \quad u=1,2, \ldots, o, \\
& x u_{u}(n)=s\left(x c_{u}(n)\right) \quad u=1,2, \ldots, o .
\end{aligned}
$$

Calculate $\alpha_{u}(n)$. The first is the error between the real output and the predicted value [22], and then calculate $\alpha_{u}(n)$ of each node of the error to the result value:

$$
\begin{aligned}
\frac{\alpha f}{\alpha v_{k u}} & =\frac{\alpha f}{\alpha x c_{u}(n)} \frac{\alpha x c_{u}(n)}{\alpha v_{k u}}, \\
\frac{\alpha x c_{u}(n)}{\alpha v_{k u}} & =\frac{\alpha\left(\sum_{k=1}^{q} v_{k u} k u_{k}(n)-p_{u}\right)}{\alpha v_{k u}}=k u_{k}(n), \\
\frac{\alpha f}{\alpha x c_{u}(n)} & =\frac{\alpha\left(1 / 2 \sum_{u=1}^{o}\left(g_{u}(n)-x u_{u}(n)\right)^{2}\right)}{\alpha x c_{u}(n)} \\
& =-\left(g_{u}(n)-x u_{u}(n)\right) x u_{u}^{\prime}(n) \\
& =-\left(g_{u}(n)-x u_{u}(n)\right) s^{\prime}\left(x c_{u}(n)\right) \cong-\beta_{u}(n) .
\end{aligned}
$$




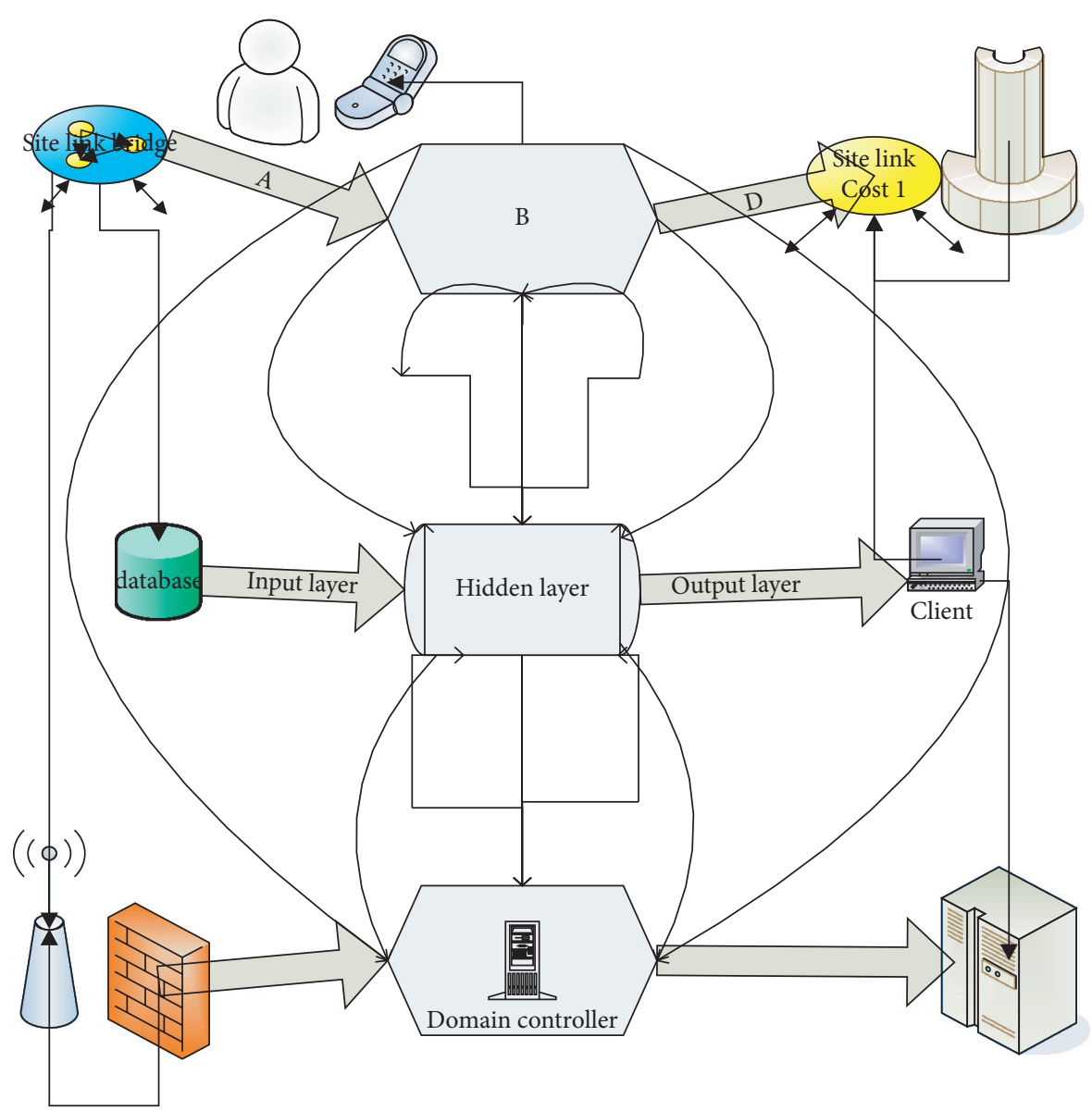

Figure 2: Feedback neural network.

Calculate $\beta_{k}(n)$, and use the connection average of the intermediate value to the result value, the output value of $\beta_{\mathrm{u}}(n)$, and the output of the intermediate value to calculate the error $\beta_{k}(n)$ for each node of the process value:

$$
\frac{\alpha f}{\alpha k c_{k}(n)}=\frac{\alpha f}{\alpha k u_{k}(n)} \frac{\alpha k u_{k}(n)}{\alpha k c_{k}(n)}=-\sum_{u=1}^{o} \beta_{u}(n) v_{k u} s^{\prime}\left(k c_{k}(n)\right) \cong-\beta_{k}(n) .
$$

Modify the connection weight $v_{k u}(n)$, and use $\beta_{u}(n)$ of each node of the output layer and the output of each node of the hidden layer to modify the connection weight $v_{k u}(n)$ :

$$
\begin{aligned}
\Delta v_{k u}(n) & =-\gamma \frac{\alpha f}{\alpha v_{k u}}=\gamma \beta_{u}(n) k u_{k}(n) \\
v_{k u}^{M+1} & =v_{k u}^{M}+\theta \beta_{u}(n) k u_{k}(n) .
\end{aligned}
$$

Modify the connection weight $v_{c k}(n)$, and use $\beta_{k}(n)$ of each node of the hidden layer and the input of each node of the input layer to modify the connection weight $v_{c k}(n)$ :

$$
v_{c k}^{M+1}=v_{c k}^{M}+\theta \beta_{k}(n) a_{c}(n) .
$$

Calculate global error:

$$
F=\frac{1}{2 w} \sum_{n=1}^{w} \sum_{u=1}^{p}\left(z_{u}(n)-x u_{u}(n)\right)^{2} .
$$

Finally, judge whether to stop learning: when the error of the BP network reaches the set error or reaches the maximum number of learning times, the algorithm ends. Otherwise, skip to the third step to continue learning [23].

Although the neural network has many advantages, it also has disadvantages. The disadvantages are mainly as follows: First of all, the process value of the neural network does not have a definite formula to guide. Secondly, because the units in each layer of the network are not connected with each other, its efficiency is also fixed, so when the module data is large, the efficiency of the neural network is directly caused to be slow. Finally, BP neural network there is often a local minimum in the overall difficulties, which does exist, so the calculation accuracy is not very high [24].

Therefore, in view of the problems and shortcomings of the above BP network, people have proposed several ways to improve; the commonly used methods are additional momentum method and adaptive learning rate method [25]. 
When the increase factor method corrects the data of the network, the scope of it is also considered on the basis of the reduction. The main method is to add a change data inversely proportional to the last weight and threshold after each weight and threshold [26]. The formula is as follows:

$$
\begin{aligned}
& \Delta v_{c l}(M+1)=(1-w s) \theta \beta_{c} a_{l}+w s \Delta v_{c l}(M) \\
& \Delta{\omega_{c}}(M+1)=(1-w s) \theta \beta_{c}+w s \Delta \omega_{c}(M) .
\end{aligned}
$$

The core idea of this method is to influence the last change of weight by adding momentum factor to act as a damper. Presence momentum factor reduced tendency of oscillation, effectively avoids the occurrence of local minima, and improves the convergence speed and accuracy [27].

The adaptive learning rate method is proposed for the problem that the learning rate of BP neural network is difficult to determine. This method provides a good solution to the problem of too large or too small learning rate, which also greatly reduces the time used for learning. Generally, if you want to converge faster, you should increase the learning rate. However, if the learning rate is too high, the network will not converge. Therefore, the formula for adaptive learning rate is proposed as follows:

$$
\theta(M+1)= \begin{cases}1.08 \theta(W) & D(W+1)<D(W) \\ 0.69 \theta(W) & D(W+1)>D(W) \times 1.02 \\ \theta(W) & \text { OTHERS }\end{cases}
$$

This method can control the learning rate to maximize under the premise of ensuring that the error meets the target requirements and achieve the fastest learning rate when the error is allowed, greatly shortening the time used for network learning.

2.3.3. RBF Neural Network Model. The RBF neural network is a model proposed by J scholars and D scholars in the early 1980s and 1990s. It is a noninteractive network with several layers. The most basic composition of the RBF network includes three layers. The starting value is some initial components; they connect the network to the world; the middle is the only process in the network, and its function is to carry out a random transformation from the initial value to the intermediate space; the result layer is line-like, which acts on the development of the initial value; providing the input/result status in response to it can use the following formula:

$$
\begin{cases}Q_{a}=u z i\left[-\frac{\left\|Z-g_{a}\right\|}{2 \alpha_{a}^{2}}\right] & a=1,2, \ldots, m \\ x=\sum_{a=1}^{m} u_{a l} q_{a}-\beta & l=1,2, \ldots, k\end{cases}
$$

Among them, $Z=(z 1, z 2, \ldots, z m)$ is the starting value, $g_{a}$ is the midpoint of the function, $\alpha_{a}$ is the normal value, which determines the width around the intermediate value, $m$ is the process level value, and $u_{a l}$ is the process value and The average of the result value node $1, \beta$ is the highest point of the output layer node, and $x$ is the output of the network [28]. Because the transfer function of the process value of the ref network uses a variable function, when the input data is close to the center range of the variable function, the intermediate data will produce a larger output, otherwise, it will produce a smaller output.

Using the data reduction method, its essence is to change the data dissemination by the principle that the mean value of the error between the input calculated value and the actual predicted data is the smallest:

The least squares error function equation is

$$
x=\frac{1}{2}\left(b(a)-b^{\prime}(a)\right)^{2}
$$

In the formula, $b(a)$ is the actual prediction and $b^{\prime}(a)$ is the current actual value of the network [29]. Data reduction is to continuously correct the average value along the negative direction of $x$ until the output error $x$ reaches the minimum.

The formula for modifying the weight of the gradient descent method is

$$
V(p+1)=V(p)+\varepsilon(p)\left(-\frac{\ell L(v)}{\ell v}\right) \mid v=v(p) .
$$

In formula (12), $\varepsilon(p)$ is the speed at which $0.1<\varepsilon<1.1$ can be changed, so that the average value can be modified.

\section{Design and Realization Method Experiment Based on the Research of Artificial Neural Network Model in Geospatial Analysis}

\subsection{Feasibility Analysis of the System}

(1) Feasibility analysis of the technical route: first, use MATLAB numerical simulation software to simulate the improved BP neural network model, and then use a compiler to convert M-under MATLAB into a callable DLL file, so that BP neural network model can be integrated in the geographic information management system. Finally, in terms of data operation and management, related technologies such as data and document databases will be used to provide relevant support to the system.

(2) Source analysis: all relevant samples needed for geology can be obtained from the bibliography of the relevant geopolitical supervision unit. This data method has relatively little money, and its reliability and accuracy are relatively high. Therefore, obtaining relevant data in this way can effectively reduce its cost and improve reliability.

(3) System operation and operation feasibility analysis: since the development process of the entire system is carried out independently by the author, considering the user's computer operation level and ability, as well as the aesthetics of the system and other issues, the author will develop a geographic information management system that is easy to operate and meets the aesthetic requirements of most users. 
3.2. System Requirement Analysis. Requirement analysis is an important work that must be carried out before the development of the system. Its work includes determining the functions to be implemented by the system, collecting the required data, and understanding the needs of users.

(1) Storage and management of geographic data include attribute data such as place name, area or local topography, and age of existence. And three-dimensional data include population density, the degree of population reeling, the terrain surrounding the population, and other related data. The main function of this geographic situation is to be able to save and manage the collected data and provide guarantees for the realization of other applications. In addition, the system must be able to update the data all the time to ensure its stability.

(2) Geographical information query and analysis: after completing the storage and management of the collected data, the system will enable users to query these data so that users can have an intuitive understanding of relevant population data, and then use these data to analyze some geographical conditions to reflect the situation of spatial data.

(3) Topographic map research: to reflect the geographic situation of a region based on relevant data, it is necessary to integrate attributes with three-dimensional data, and then make corresponding maps through the data provided by the network.

(4) Geospatial analysis: through the past data and information, the improved BP neural network model is used to enable users to have an intuitive understanding of the future geographic development, and then use it as a powerful basis for formulating related geographic spaces.

For example, the function contains rich data information. The composition and structure of the geology and the liquid-filled nature of the interval affect the difference of observations. Therefore, the logging curves of various parameters related to rocks are the basis of lithology analysis. The logging curves for rock robustness include ordinary potential, ordinary current, and resistance. Select ordinary current and resistivity to explore lithology.

When using data values to identify geological lithology, firstly, based on the corresponding characteristics of the rock samples and parameters in the area, the lithology of the rock samples in the classification area is divided into luminescent limestone, silty sandstone, and granular limestone. Extract the parameter values corresponding to some samples from various types of rocks, determine the interaction relationship between lithology and parameter values, see Table 1, and then create the corresponding model through the program.

It can be seen from Table 1 that the algorithm system has the function of automatic adjustment and can classify the collected samples; it has strong operability and high resolution of the samples; research shows that the application of the model for data lithology identification is achievable.
TABle 1: Correspondence table of SOFM network lithology recognition model.

\begin{tabular}{lccccccc}
\hline Number & api & Us/m & $\mathrm{mv}$ & $\mathrm{cm}$ & $\mathrm{lml}$ & $\mathrm{nml}$ & Lithology \\
\hline $\mathrm{A}$ & 21.3 & 160.1 & 23.3 & 20.9 & 9.7 & 10.3 & Fluoresce \\
$\mathrm{B}$ & 24.9 & 165.3 & 26.1 & 22.3 & 9.2 & 9.1 & Limestone \\
$\mathrm{C}$ & 113.9 & 203.1 & 69.3 & 23.6 & 7.9 & 8.1 & Siltstone \\
$\mathrm{D}$ & 128.6 & 206.5 & 71.4 & 24.1 & 7.8 & 8.6 & Argilla \\
$\mathrm{E}$ & 64.3 & 174.9 & 51.8 & 19.8 & 5.8 & 9.6 & Gluten \\
$\mathrm{F}$ & 59.9 & 171.3 & 51.8 & 19.1 & 4.3 & 9.1 & Clastic limestone \\
\hline
\end{tabular}

\section{Experimental Results Based on the Design and Realization Method of Artificial Neural Network Model in Geospatial Analysis}

4.1. Survey Content and Sample Analysis. According to the comprehensive information provided by a province, determining the content of the gold deposits in a distribution of observed target province, the network model is used to study the distribution of gold deposits in the abnormal area unit of the province. The results show that the development of gold has some relevance, providing some data to support the gold mining province.

It can be seen from Figure 3 that the division method is used to divide the whole. According to the gold distribution area of the province, a total of 100 deposit concentration areas and abnormal concentration area units are divided, of which there are 5 deposit concentration area model units, numbered $a, b, \ldots, e, 75$ gold anomaly concentrated areas, the numbers are 001, 002, .., 089; see Table 2 .

Among them, the definite value is assigned to the definite value, and the logical value is assigned to the grade evaluation result that has no definite value and only a logical relationship. The logical value adopts a binary variable, which is $x$ for existence and $y$ for nonexistence. See Table 2 for details.

According to the parameters in Table 2, in order to explore the network, the data of 10 gold deposit concentration area units of known divisions are selected as the initial training mode of the BP network, as shown in Table 2. The range of each gold deposit concentration area unit is used as the target result model of training. The size of the deposit can be divided into large, medium, and small units, as shown in Figure 4.

It can be seen from Figure 4 that after the exercise is completed, the user can click on the "Deflection Degree" option to enter the error curve to view the error reduction of this exercise. Use practice mode to collect data to train the network model to determine the average value and fault of the network. After the exercise is successful, the initial layer of the initial network of the prediction sample, the initial value is broadcast from the initial layer through the middle layer to the final layer, and the predicted value of the BP network is calculated.

4.2. Application of the Model. In order to test the stability of the geological evaluation model function and the reliability of the operating results, the data of two counties are selected 
Concentrated area of ore deposits

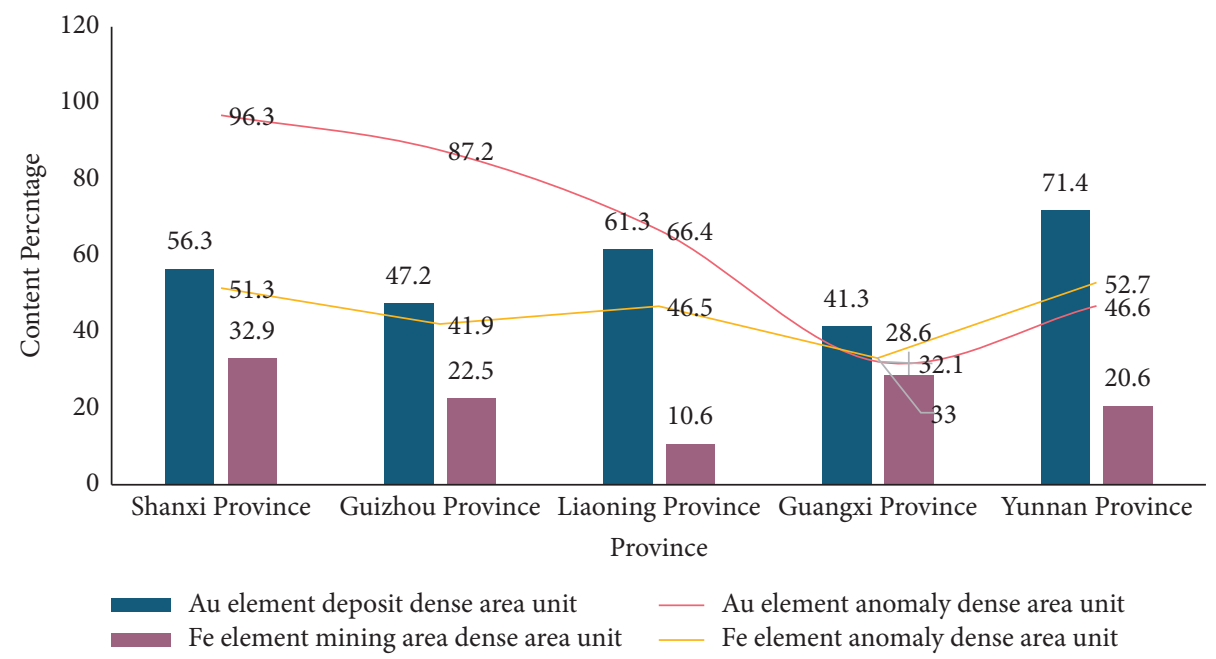

FIGURE 3: Unit map of the intensive and abnormal intensive areas of mineral deposits in various provinces.

TABle 2: Model unit (training sample) data table.

\begin{tabular}{|c|c|c|c|c|c|c|c|}
\hline $\begin{array}{l}\text { Unite } \\
\text { number }\end{array}$ & $\begin{array}{l}\text { Percentage of abnormal } \\
\text { area in unit area }\end{array}$ & $\begin{array}{l}\text { Associated and symbiotic } \\
\text { elemental land number } \\
\text { Fe } \mathrm{Cu} \mathrm{Pb} \mathrm{Zn} \mathrm{Hg} \mathrm{Ag}\end{array}$ & $\begin{array}{c}\text { Base } \\
\text { assignment }\end{array}$ & $\begin{array}{l}\text { Neutral acid } \\
\text { value }\end{array}$ & $\begin{array}{c}\text { Base } 2 \\
\text { level }\end{array}$ & $\begin{array}{l}\text { Aeromagnetic } \\
\text { level } 1\end{array}$ & $\begin{array}{l}\text { Permian } \\
\text { assignment }\end{array}$ \\
\hline$a$ & 11.3 & 85147 & $x$ & $z$ & $y$ & $x$ & 1.1 \\
\hline$b$ & 1.48 & 45122 & $x$ & $x$ & $x$ & $x$ & 2.4 \\
\hline$c$ & 5.24 & 79487 & $y$ & $z$ & $y$ & $y$ & 0.3 \\
\hline$d$ & 3.63 & 64853 & $x$ & $z$ & $x$ & $y$ & 1.6 \\
\hline$e$ & 1.08 & 481094 & $x$ & $y$ & $y$ & $x$ & 2.1 \\
\hline
\end{tabular}

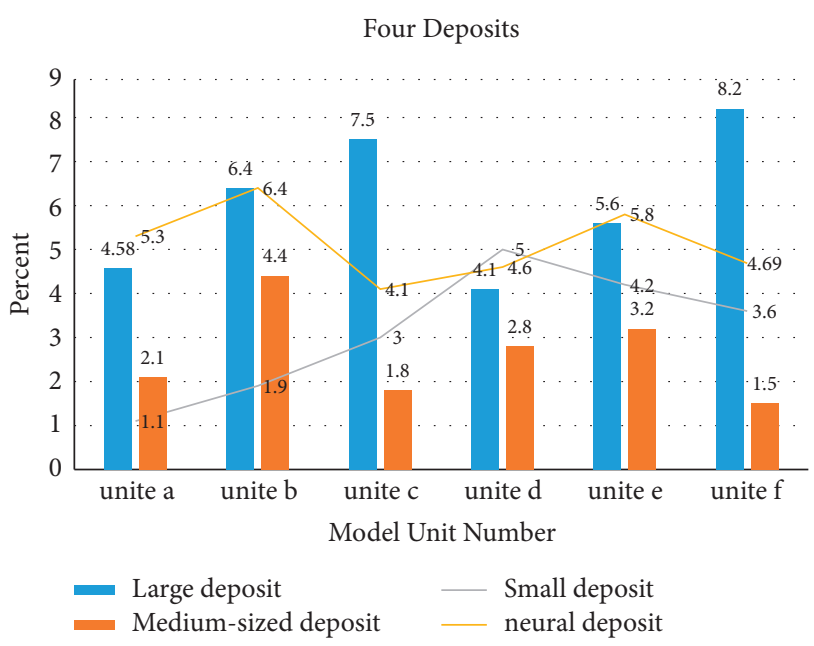

FIGURE 4: BP network training target model diagram.

as examples of the geological disaster model. These two counties have done geohazard-related cases. The data from County A is used to test the model, and the data from County B is used to ensure that the model is reliable. The geographic form of County A is dominated by sandy land. The landform in County B is dominated by plains, with small flatlands and low mountains running through hills.
Contemporary geology is distributed in hills, small river basins, and some shoals; Cretaceous strata are located in Xiaopingdi, southerly hills and shoal sand dunes in County $\mathrm{B}$; shallow hilly areas to the west and south of B and low mountain areas in the east of A. It is the Jurassic strata. The local characteristics of each layer are shown in Table 3.

It can be seen from Table 3 that the main types of disasters in the region are mudslides, collapses, and crumbling slopes. After investigation, 61 geological points were discovered in County A, of which 47 were mudslides, 4 collapses, and 10 crumbling slopes. The mudslides were the main ones, and the scale was mainly small- and mediumsized. Disasters are mainly distributed in 5 towns in the eastern low mountain area; 19 geological disasters occurred in County B, including 9 shaky slopes, 6 mudslides, and 4 collapses. Shaky slopes are the main types in the county, and the types are mainly small-scale. Disasters are mainly distributed in the low mountains in the south.

Using the collected data of County A, the altitude, cutting depth, geological disaster status layer, and the prediction result layer of the easily causing area were compiled according to the image expression. The evaluation factor layer after making is shown in Figure 5.

It can be seen from Figure 5 that through the extraction function of the model analysis of the geological disaster information system, select the sampled altitude, cutting 
TABLE 3: Summary table of lithology description in the study area.

\begin{tabular}{|c|c|c|c|c|}
\hline \multicolumn{2}{|c|}{ Stratum name } & $\begin{array}{l}\text { Code } \\
\text { name }\end{array}$ & Distributed & Lithology \\
\hline \multirow{3}{*}{$\begin{array}{l}\text { Fourth } \\
\text { series }\end{array}$} & Holocene & $a$ & $\begin{array}{l}\text { Both sides of the river, first-level terraces, and } \\
\text { floodplains }\end{array}$ & $\begin{array}{l}\text { The upper section is mainly brown-red, } \\
\text { brown-yellow silty clay }\end{array}$ \\
\hline & $\begin{array}{l}\text { Upper } \\
\text { Pleistocene } \\
\text { system }\end{array}$ & $b$ & Plain areas, secondary terraces, and some terrace areas & The upper part is gray-yellow sandy clay \\
\hline & Pleistocene & $c$ & $\begin{array}{c}\text { There is also a small amount of sporadic exposure in } \\
\text { the plateau area and the plain area }\end{array}$ & The upper part is brown-red sandy clay \\
\hline \multirow{2}{*}{ Cretaceous } & Irrigation group & $d$ & $\begin{array}{l}\text { Exposed in the eastern Taiwan region of County B, } \\
\text { sporadically exposed on the two wings of the su wharf } \\
\text { anticline in County A }\end{array}$ & $\begin{array}{l}\text { Brown-red mudstone with argillaceous } \\
\text { siltstone }\end{array}$ \\
\hline & Clipping group & $e$ & $\begin{array}{l}\text { Exposed in the low mountains in the southern part of } \\
\text { county B and the two wings of the su wharf anticline in } \\
\text { County A }\end{array}$ & $\begin{array}{l}\text { Brown-red, gray-yellow, medium-to- } \\
\text { thick massive silty fine sandstone, } \\
\text { argillaceous siltstone }\end{array}$ \\
\hline \multirow[t]{2}{*}{ Jurassic } & $\begin{array}{l}\text { Penglai town } \\
\text { group }\end{array}$ & $f$ & The shallow hilly area in the south of County B & $\begin{array}{l}\text { The upper part is purple-red, purple- } \\
\text { grey medium-thick layered fine } \\
\text { sandstone }\end{array}$ \\
\hline & Suining group & $g$ & $\begin{array}{l}\text { North west wing of Longquanshan anticline, Shuangliu } \\
\text { county }\end{array}$ & $\begin{array}{l}\text { Fuchsia mudstone intercalated with } \\
\text { argillaceous siltstone }\end{array}$ \\
\hline
\end{tabular}

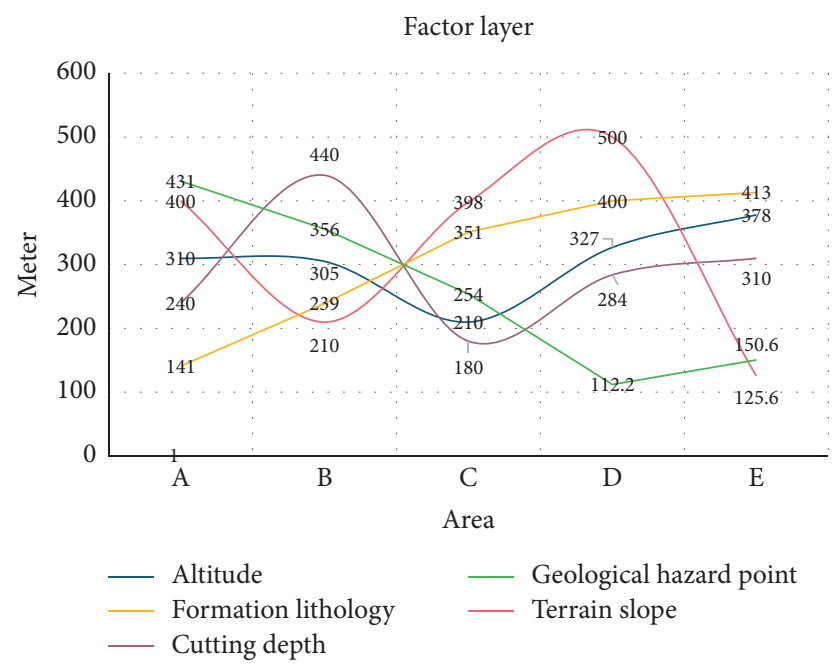

Figure 5: Evaluation factor layer of a county.

depth, the current status of the geological disaster, and the final layer of the expected output, and then complete each layer in turn according to the guidance. The data is extracted and finally output as a database. The calculation method is to set different speeds according to a certain increment value to obtain the corresponding error value. The setting range of the speed value is generally between 0.1 and 9, and the increment value is 0.3 . After the training is completed, make the recorded speed value and the corresponding error into the training error comparison table in Table 4 and the training error analysis chart in Figure 6.

As can be seen from Table 4, Figure 6 is the training error analysis diagram.

It can be clearly found from Figure 6 that when the expansion speed of the network model is $\geq 1.0$, the corresponding error curve begins to be straight and flat. Therefore, here we take the value of speed $=1.3$ at the inflection point of the curve as the training parameter of the final network model to complete the training of the network model. Figure 7 is the training curve of the software when speed is 1.3 .

It can be seen from Figure 7 that after the evaluation model training is completed, the data of County B, which has similar geological environmental conditions as County A, and the network model parameters completed in the previous training are used to generate geohazard-prone areas, and then the generated geohazard-prone areas perform overlay analysis with actual surveyed geological hazards to verify the reliability of the model. We have made statistics on the number of geological hazard points in different levels of prone areas, and the statistical results are shown in Table 5.

From the analysis in Figure 7 and Table 5, it can be seen that the geological disaster points are distributed in the prone areas, accounting for $99.7 \%$, and the disasters in the 
TABLE 4: Comparison table of training error of different spread values.

\begin{tabular}{lccccccc}
\hline Expansion speed & Error & Expansion speed & Error & Expansion speed & Error & Expansion speed & Error \\
\hline 0.1 & 0.544564 & 2.2 & 0.195441 & 4.3 & 0.137651 & 6.4 & 0.124758 \\
0.4 & 0.482154 & 2.5 & 0.154421 & 4.6 & 0.136241 & 6.7 & 0.120131 \\
0.7 & 0.294567 & 2.8 & 0.148721 & 4.9 & 0.129784 & 7.0 \\
1.0 & 0.156471 & 3.1 & 0.165877 & 5.2 & 0.127845 & 0.120124 \\
1.3 & 0.124835 & 3.4 & 0.146795 & 5.5 & 0.129647 & \\
1.6 & 0.164897 & 3.7 & 0.148759 & 5.8 & 0.126547 & \\
1.9 & 0.194541 & 4.0 & 0.134587 & 6.1 & 0.128457 & \\
\hline
\end{tabular}

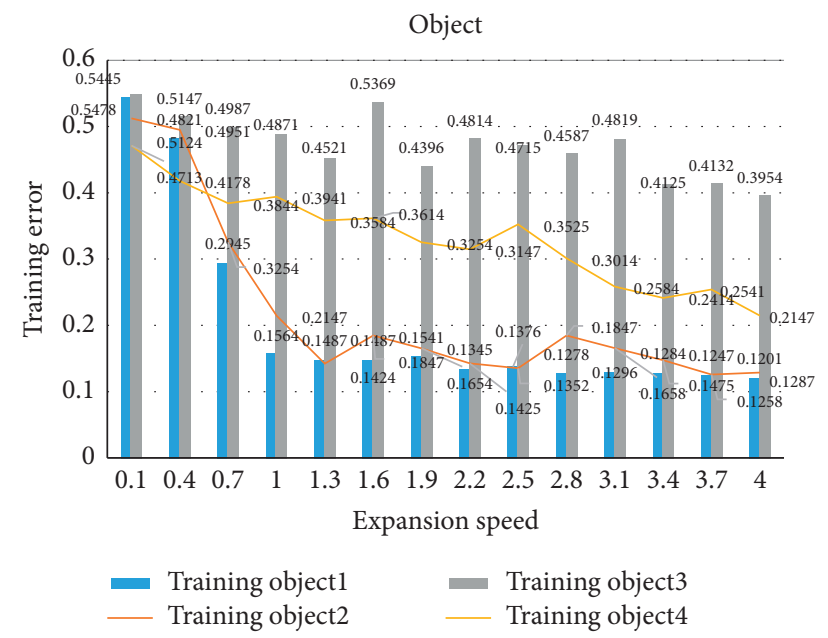

Figure 6: Training error analysis diagram of different spread values.

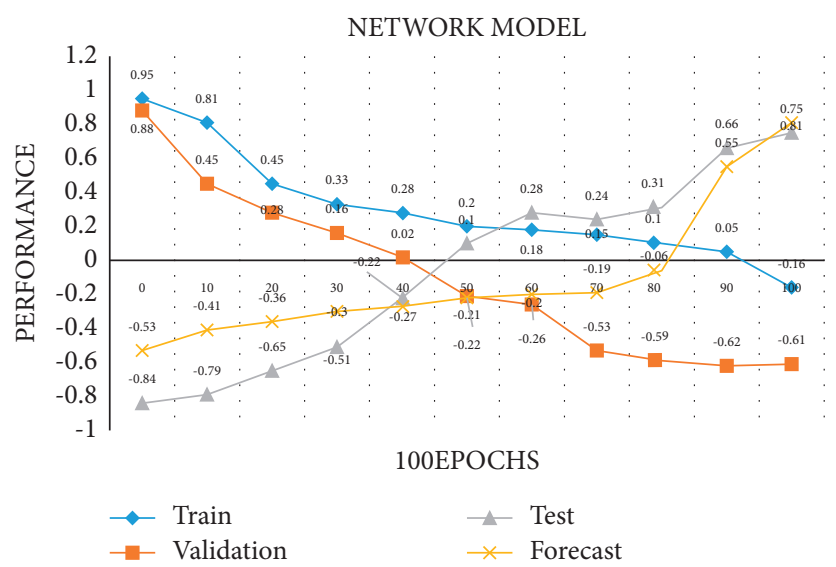

Figure 7: RBF network model training curve.

TABLE 5: Statistics on the number of geological hazards in different grade areas.

\begin{tabular}{lcccc}
\hline & High-prone area & Central prone area & Low-prone zone & Less prone area \\
\hline Evaluation zone & 11 & 4 & 5.5 & 0.1 \\
The proportion (\%) & 64.5 & 12.4 & 22.8 & 0.3 \\
Risk assessment (\%) & 97 & 28 & 58 & 4 \\
Reliability & Quite reliable & Generally reliable & Low reliability & The number of samples is too small \\
\hline
\end{tabular}

nonprone areas are less distributed, with a proportion of $0.3 \%$, which is in line with the real situation of geological disaster points in the suburbs. Moreover, in the geological disaster-prone areas, the proportion of geological disasters in the high-risk and moderate-prone areas accounted for $76.9 \%$, and the number of disaster points in the low-level 
prone areas accounted for $22.8 \%$. It is basically in line with the actual level and can meet the needs of geography-related work.

\section{Conclusions}

This article discusses the research process of neural network and its book guidance. For the function graph of the network, the data classification method is used to give a simple and cautious expression. On the basis of extensive research on BP algorithm, I chose algorithm and completed the program. Using the analytic hierarchy process, the information analysis method and the artificial network analysis method, samples were collected, the current bottleneck of the artificial network was analyzed, and the algorithm was simplified. And create an old model. In the investigation of the system of A and B counties, 61 geological disasters were found in County A, of which 47 were mudslides, 4 collapses, and 10 slopes. There were 19 geological disasters in County B, including 9 slopes, 6 mudslides, and 4 collapses. Comparing with the new model, it is shown that the geological disasters are all distributed in the areas prone to geo-geological disasters, with a ratio of $99.7 \%$. In the nonprone areas, the geographic distribution is less, with a ratio of $0.3 \%$. Moreover, the high-prone areas and the mediumprone areas, the proportion of suffering in the area accounted for $76.9 \%$, and the number of suffering points in the low-prone area accounted for $22.8 \%$. The geographical and geological grades divided by the evaluation model are basically consistent with the actual grades, which can meet the needs of the geographical evaluation work. The shortcomings of this paper are as follows: first, the original neural network algorithm is improved in this subject, but the improved method of this subject does not significantly improve some special data, and the neural network model has room for improvement; secondly, the project requirements of the project and some collection forms are uncertain, and the supervision indicators are not clear enough, so that some operation procedures and database design are not concise and even cumbersome. Therefore, in the simplification and refinement of future models, we can specifically simplify and standardize data collection methods for specific data, simplify the process, and make the artificial neural network model have profound operability in the geographic information system.

\section{Data Availability}

The data that support the findings of this study are available from the corresponding author upon reasonable request.

\section{Conflicts of Interest}

The authors declare no potential conflicts of interest with respect to the research, authorship, and/or publication of this article.

\section{Acknowledgments}

This study was supported by the Key Research Program of the Innovation Academy for Green Manufacture, Chinese Academy of Sciences (IAGM-2019-A16), and the National
Social Science Foundation of China (18VDL010), and the Alliance of International Science Organizations (ANSO-CRKP-2020-02).

\section{References}

[1] B. Li, Y. F. Zeng, B. B. Zhang, and X. Q. Wang, "A risk evaluation model for karst groundwater pollution based on geographic information system and artificial neural network applications," Environmental Geology, vol. 77, no. 9, pp. 344.1-344.14, 2018.

[2] R. Arabgol, M. Sartaj, and K. Asghari, "Predicting nitrate concentration and its spatial distribution in groundwater resources using support vector machines (SVMs) model," Environmental Modeling and Assessment, vol. 21, no. 1, pp. 71-82, 2016.

[3] I. Ilia and P. Tsangaratos, "Applying weight of evidence method and sensitivity analysis to produce a landslide susceptibility map," Landslides, vol. 13, no. 2, pp. 379-397, 2016.

[4] M. Yousefi and E. J. M. Carranza, "Data-driven index overlay and boolean logic mineral prospectivity modeling in greenfields exploration," Natural Resources Research, vol. 25, no. 1, pp. 3-18, 2016.

[5] D. Tang, F. Gharagheizi, and D. S. Sholl, "Adsorption-based separation of near-azeotropic mixtures-A challenging example for high-throughput development of adsorbents," The Journal of Physical Chemistry B, vol. 125, no. 3, pp. 926-936, 2021.

[6] H. Bourenane, M. S. Guettouche, Y. Bouhadad, and B. Massinissa, "Landslide hazard mapping in the Constantine city, Northeast Algeria using frequency ratio, weighting factor, logistic regression, weights of evidence, and analytical hierarchy process methods," Arabian Journal of Geosciences, vol. 9, no. 2, pp. 1-24, 2016.

[7] A. Suleiman, M. R. Tight, and A. D. Quinn, "Hybrid neural networks and boosted regression tree models for predicting roadside particulate matter," Environmental Modeling and Assessment, vol. 21, no. 6, pp. 731-750, 2016.

[8] M. J. Lee, I. Park, J. S. Won, and S. Lee, "Landslide hazard mapping considering rainfall probability in Inje, Korea," Geomatics, Natural Hazards and Risk, vol. 7, no. 1, pp. 424446, 2016.

[9] S. Kariminia, S. Motamedi, S. Shamshirband, D. Petković, C. Roy, and R. Hashim, "Adaptation of ANFIS model to assess thermal comfort of an urban square in moderate and dry climate," Stochastic Environmental Research and Risk Assessment, vol. 30, no. 4, pp. 1189-1203, 2016.

[10] J. Li, X. Tian, W. Huang, B. Zhang, and S. Fan, "Application of long-wave near infrared hyperspectral imaging for measurement of soluble solid content (SSC) in pear," Food Analytical Methods, vol. 9, no. 11, pp. 3087-3098, 2016.

[11] A. Mansouri, A. Nazari, and M. Ramazani, "A comparison of artificial neural network model and logistics regression in prediction of companies' bankruptcy (a case study of Tehran stock exchange)," International Journal of Advanced Computer Research, vol. 6, no. 24, pp. 81-92, 2016.

[12] E. Funes, Y. Allouche, G. Beltrán, M. P. Aguliera, and A. Jiménez, "A predictive artificial neural network model as a simulator of the extra virgin olive oil elaboration process," Journal of Near Infrared Spectroscopy, vol. 25, no. 4, pp. 278-285, 2017.

[13] B. Grandjean and M. A. Maier, "Emergence of gamma motor activity in an artificial neural network model of the corticospinal system," Journal of Computational Neuroscience, vol. 42, no. 1, pp. 53-70, 2017. 
[14] J. Y. Charati, G. Janbabaei, N. Alipour, S. Mohammadi, S. Ghorbani, and A. Fendereski, "Survival prediction of gastric cancer patients by artificial neural network model," Gastroenterology and Hepatology from Bed to Bench, vol. 11, no. 2, pp. 110-117, 2018.

[15] A. G. Banhatti and P. C. De Ka, "Effects of data pre-processing on the prediction accuracy of artificial neural network model in hydrological time series," Journal of Business Ethics, vol. 34, no. 2, pp. 101-121, 2016.

[16] I. Butun, P. Osterberg, and H. Song, "Security of the internet of things: vulnerabilities, attacks, and countermeasures," IEEE Communications Surveys and Tutorials, vol. 22, no. 1, pp. 616-644, 2020.

[17] O. D. Taratuhin, L. Y. Novikova, I. V. Seferova et al., "An artificial neural network model to predict the phenology of early-maturing soybean varieties from climatic factors," Biophysics, vol. 65, no. 1, pp. 106-117, 2020.

[18] C. Rymab, C. Hzlma, B. Tao et al., "Artificial neural network model for preoperative prediction of severe liver failure after hemihepatectomy in patients with hepatocellular carcinoma," Surgery, vol. 168, no. 4, pp. 643-652, 2020.

[19] Z. Lv, H. Song, P. Basanta-Val, A. Steed, and M. Jo, "Nextgeneration big data analytics: state of the art, challenges, and future research topics," IEEE Transactions on Industrial Informatics, vol. 13, no. 4, pp. 1891-1899, 2017.

[20] S. Velazquez Medina and U. Portero Ajenjo, "Performance improvement of artificial neural network model in short-term forecasting of wind farm power output," Journal of Modern Power Systems and Clean Energy, vol. 8, no. 3, pp. 484-490, 2020.

[21] D. Guido, H. B. Song, and S. Anke, Big Data Analytics for Cyber-Physical Systems: Machine Learning for the Internet of Things, pp. 1-360, Elsevier, Amsterdam, Netherlands, 2019.

[22] M. O. Selbesoglu, "Prediction of tropospheric wet delay by an artificial neural network model based on meteorological and GNSS data," Engineering Science and Technology, vol. 23, no. 5, pp. 967-972, 2020.

[23] V. K. H. Chan and C. W. Chan, "Towards explicit representation of an artificial neural network model: comparison of two artificial neural network rule extraction approaches," Petroleum, vol. 6, no. 4, pp. 329-339, 2020.

[24] J. Chang, G. Wang, and L. Guo, "Simulation of soil thermal dynamics using an artificial neural network model for a permafrost alpine meadow on the Qinghai-Tibetan plateau," Permafrost and Periglacial Processes, vol. 30, no. 3, pp. 195207, 2019.

[25] Z. H. Wani and S. M. K. Quadri, “An improved particle swarm optimisation-based functional link artificial neural network model for software cost estimation," International Journal of Swarm Intelligence, vol. 4, no. 1, pp. 38-54, 2019.

[26] H. B. Song, G. A. Fink, and J. Sabina, Security and Privacy in Cyber-Physical Systems: Foundations, Principles and Applications, pp. 1-472, Wiley-IEEE Press, Chichester, UK, 2017.

[27] W. M. Jung, I. S. Park, Y. S. Lee et al., "Characterization of hidden rules linking symptoms and selection of acupoint using an artificial neural network model," Frontiers of Medicine, vol. 13, no. 1, pp. 112-120, 2019.

[28] F. Fariba Safa, A. A. Dakhel, and S. Shariati, "Predictive artificial neural network model for solvation enthalpy of organic compounds in N,N-dimethylformamide," Russian Journal of Physical Chemistry, vol. 93, no. 13, pp. 2661-2668, 2019.

[29] K. Vonsevych, M. F. Goethel, J. Mrozowski, J. Awrejcewicz, and M. Bezuglyi, "Fingers movements control system based on artificial neural network model," Radioelectronics and Communications Systems, vol. 62, no. 1, pp. 23-33, 2019. 